\title{
Contributions from SUSY-FCNC couplings to the interpretation of the HyperCP events for the decay $\Sigma^{+} \rightarrow p \mu^{+} \mu^{-}$
}

\author{
Gao Xiangdong:* Chong Sheng Lit Z Zhao Li, $\$$ and Hao Zhang $\$$ \\ Department of Physics, Peking University, Beijing 100871, China
}

(Dated: November 21, 2018)

\begin{abstract}
The observation of three events for the decay $\Sigma^{+} \rightarrow p \mu^{+} \mu^{-}$with a dimuon invariant mass of $214.3 \pm 0.5 \mathrm{MeV}$ by the HyperCP collaboration imply that a new particle $\mathrm{X}$ may be needed to explain the observed dimuon invariant mass distribution. We show that there are regions in the SUSY-FCNC parameter space where the $A_{1}^{0}$ in the NMSSM can be used to explain the HyperCP events without contradicting all the existing constraints from the measurements of the kaon decays, and the constraints from the $K^{0}-\bar{K}^{0}$ mixing are automatically satisfied once the constraints from kaon decays are satisfied.
\end{abstract}

PACS numbers: 14.80.Cp, 12.60.Jv, 14.20.Jn

*Electronic address: gaoxiangdong@pku.edu.cn

${ }^{\dagger}$ Electronic address: csli@pku.edu.cn

${ }^{\ddagger}$ Electronic address: zhli.phy@pku.edu.cn

§Electronic address: haozhang.pku@pku.edu.cn 


\section{INTRODUCTION}

Recently, there are a great deal of interest [1, 2, 3, 4, 5, 6, 7, 8, 9, 10] in the interpretation of the observed three events for the decay $\Sigma^{+} \rightarrow p \mu^{+} \mu^{-}$with a dimuon invariant mass of $214.3 \pm 0.5 \mathrm{MeV}$, which were reported by the HyperCP Collaboration [11]. The branching ratio based on the three events for this process is $\left(8.6_{-5.4}^{+6.6} \pm 5.5\right) \times 10^{-8}$ [11]. It has been argued that in the framework of the Standard Model(SM) it is possible to account for the total branching ratio when the long-distance contributions are properly included, but all the three events are around $214 \mathrm{MeV}$ cannot be explained [11, 12]. If no new evidence support the SM explanations in the future experiments with more events, it is most likely to interpret the three events with the existence of a new particle, X, beyond the SM. However, a new particle explanation for the HyperCP events seems too radical because there are not earlier experiments which observe such a $214 \mathrm{MeV}$ new particle. If this new light particle does indeed exists and contributes to the hyperon decay, it may also contribute to the kaon and B-meson decays. So, the fact that lots of experiments at this low-energy region did not observe the new $214 \mathrm{MeV}$ particle means that strong constraints has been imposed on the new particle explanation for the HyperCP events.

The authors of Ref. [8] proposed a argument to explain the HyperCP events for the hyperon decay with the new particle $\mathrm{X}$ without contradicting with the constraints on the $\mathrm{X}$ from the low-energy experiments. As shown in Ref. [8], in addition to the flavor-changing two-quark contributions, there are also four-quark contributions arising from the combined effects of the usual SM $|\Delta S|=1$ operators and the flavor-conserving couplings of $\mathrm{X}$, which are comparable with the two-quark ones and cancel sufficiently to lead to suppressed rare kaon decays rates while combining the above two kinds of contributions yields $\Sigma \rightarrow p \mu^{+} \mu^{-}$ rates within the required bounds.

Based on the analysis in Ref. [8], the authors of Ref. [7] pointed out that a light pseudoscalar Higgs particle $A_{1}^{0}$ in the next-to-minimal supersymmetric standard model (NMSSM) [13, 14, 15] can be identified with $\mathrm{X}$. In fact, the mass of the light pseudoscalar Higgs particle $A_{1}^{0}$ in the NMSSM can be as small as $214 \mathrm{MeV}$ in the large-tan $\beta$ limit. Under some assumptions, it has been shown in Ref. [7] that there are regions in the parameter space 
where $A_{1}^{0}$ can satisfy the following constraints:

$$
\begin{aligned}
\mathcal{B}\left(K^{ \pm} \rightarrow \pi^{ \pm} A_{1}^{0}\right) & \lesssim 8.7 \times 10^{-9} \\
\mathcal{B}\left(K_{s} \rightarrow \pi^{0} A_{1}^{0}\right) & \lesssim 1.8 \times 10^{-9} \\
\mathcal{B}\left(B \rightarrow X_{s} A_{1}^{0}\right) & \lesssim 8.0 \times 10^{-7}
\end{aligned}
$$

which are obtained in Ref. [8] from the measurements of the kaon and B-meson decays [16, 17, 18, 19, 20], and simultaneously explain the HyperCP events.

However, the author of Ref. [7] only considered contributions from the SUSY charged current, i.e.,contributions arising from the exchanges of chargino and squark, and do not include contributions arising from SUSY-flavor-changing neutral currents(FCNC). It is well known that the SUSY-FCNC couplings can yield important(and, sometimes, even dominate) contributions to low-energy flavor physics, so further investigation on the possibility of the SUSY-FCNC mediating HyperCP events is needed. In this paper, we show that the SUSYFCNC effects also can explain the HyperCP events and satisfy all the constraints in Eq.(1). We adopt the mass insertion method [21, 22, 23, 24] to parameterize the flavor-changing effects and calculate SUSY-FCNC contributions to branching ratio of $\Sigma \rightarrow p A_{1}^{0}$ and rare kaon decays. This method introduce the super-CKM basis for the quark and squark states. The couplings of quarks and squarks to the neutral gauginos are flavor diagonal, while the flavorchanging SUSY effects are exhibited in the off-diagonal terms of the squark mass matrix denoted by $\left(\Delta_{i j}^{q}\right)_{I J}$, where $I, J=L, R$ and $i, j=1,2,3$ indicate chiral and flavor indices respectively, and $q=u, d$ denote the type of quark. The squark propagator is then expanded as a series of $\left(\delta_{i j}^{q}\right)_{I J}=\left(\Delta_{i j}^{q}\right)_{I J} / \tilde{m}^{2}$, where $\tilde{m}$ is an average squark mass. Using the mass insertion method, we can perform calculations of the SUSY-FCNC contributions to $\Sigma \rightarrow p A_{1}^{0}$ and rare kaon decays. Since the relevant $\left(\delta_{i j}^{q}\right)_{I J}$ does not involve in the B-mesons decay, we do not consider the constraints from B-mesons decay. It is well known[23, 24, 25, 26, 27, 28, 29] that the parameters $\left(\delta_{12}^{d}\right)_{I J}$ used in our calculations also yield important contributions to the $K^{0}-\bar{K}^{0}$ mixing, however, our calculations will show that the measurements of the $K_{L}-K_{S}$ mass difference and the indirect $\mathrm{CP}$ violation observable $\epsilon_{K}$ do not lead to more stringent constraints than ones from kaon decays.

We organize our paper as follows. In Sec. II we give a brief summary of the NMSSM. In Sec. III we calculate the two-quark flavor-changing contributions to the $\Sigma$ and kaon decays arising from the SUSY-FCNC effects mediated by neutralino and gluino. In Sec. IV] 
we combine our two-quark contributions with four-quark contributions in Ref.[8] to give a numerical results and discussion. Feynman rules and analytical expressions for the fourquark contributions are collected in the Appendix A and B, respectively.

\section{NMSSM}

In order to make our paper self-contained, we start with a brief description of the NMSSM and the relevant couplings considered in our paper. The superpotential of the NMSSM is given by [13, 14, 15]

$$
W=Q Y_{u} H_{u} U+Q Y_{d} H_{d} D+L Y_{e} H_{d} E+\lambda H_{d} H_{u} N-\frac{1}{3} k N^{3},
$$

where $H_{u}$ and $H_{d}$ are the $S U(2)$ doublet with the hypercharge $1 / 2$ and $-1 / 2$ and are responsible for the up- and down-type quark mass, respectively. The ratio of the vacuum expectation values(VEVs) of the $H_{u}$ and $H_{d}$ is defined as $\tan \beta$, which are just like those in the the minimal supersymmetric standard model(MSSM). Compared with the MSSM, there is one more gauge-singlet Higgs Field $N$ with the hypercharge 0 and VEV $x$ in the NMSSM. After breaking of the supersymmetry, there are seven physical Higgs bosons in the NMSSM, including two charged Higgs bosons, three neutral scalar and two pseudoscalar Higgs bosons.

The Higgs potential of NMSSM is [30]

$$
V_{\text {Higgs }}=V_{\text {soft }}+V_{F}+V_{D}
$$

where

$$
\begin{aligned}
V_{\text {soft }} & =m_{H_{d}}^{2}\left|H_{d}\right|^{2}+m_{H_{u}}^{2}\left|H_{u}\right|^{2}-\left(\lambda A_{\lambda} H_{d} H_{u} N-\frac{1}{3} k A_{k} N^{2}+\text { H.c. }\right), \\
V_{F} & =|\lambda|^{2}\left(\left|H_{d}\right|^{2}+\left|H_{u}\right|^{2}\right)|N|^{2}+\left|\lambda H_{d} H_{u}-k N^{2}\right|^{2} \\
V_{D} & =\frac{g^{2}+g^{\prime 2}}{8}\left(\left|H_{d}\right|^{2}-\left|H_{u}\right|^{2}\right)+\frac{g^{2}}{2}\left|H_{u}^{\dagger} H_{d}\right|^{2} .
\end{aligned}
$$

The above Higgs potential has a global $U(1)_{R}$ symmetry in the limit of vanishing parameters $A_{k}, A_{\lambda} \rightarrow 0[31]$. If the global $U(1)_{R}$ symmetry is broken slightly, the lighter pseudoscalar $A_{1}^{0}$ has a natural small mass:

$$
m_{A_{1}^{0}}^{2}=3 k x A_{\lambda}+\mathcal{O}\left(\frac{1}{\tan \beta}\right)
$$


In the large $\tan \beta$ limit, $m_{A_{1}^{0}}$ can be as low as $\sim 100 \mathrm{MeV}[30]$ [31].

The Lagrangian describing the couplings of $A_{1}^{0}$ to the up- and down-type quarks and to the leptons are given by [7]

$$
\mathcal{L}_{\mathcal{A} q q}=-\left(l_{u} m_{u} \bar{u} \gamma_{5} u+l_{d} m_{d} \bar{d} \gamma_{5} d\right) \frac{i A_{1}^{0}}{v}
$$

and

$$
\mathcal{L}_{\mathcal{A} \ell}=\frac{i g_{\ell} m_{\ell}}{v} \bar{\ell} \gamma_{5} \ell A_{1}^{0}
$$

respectively, where

$$
l_{d}=-g_{\ell}=\frac{v}{\sqrt{2} x}\left(\frac{A_{\lambda}-2 k x}{A_{\lambda}+k x}\right), \quad l_{u}=\frac{l_{d}}{\tan ^{2} \beta} .
$$

Note that $l_{u}$ can be neglected in the large-tan $\beta$ limit. The four-quark contributions can be deduced from the interactions in Eq. (6) combined with the operators due to W exchange between quarks [7].

It has been shown in Ref. [7] that an $A_{1}^{0}$ of mass $214.3 \mathrm{MeV}$ decay dominantly to muonantimuon pair, and $\mathcal{B}\left(A_{1}^{0} \rightarrow \mu^{+} \mu^{-}\right) \sim 1$ can be assumed. In addition, the constraint imposed by the muon anomalous magnetic moment is given by [4]

$$
\left|g_{\ell}\right| \lesssim 1.2
$$

Moreover, the neutralino sector of the NMSSM is different from that in the MSSM. There are five neutralinos in the NMSSM, and the Lagrangian for the mass term of the neutralinos can be written as 15$]$

$$
\mathcal{L}_{m_{\chi^{0}}}=-\frac{1}{2}\left(\psi^{0}\right)^{T} Y \psi^{0}+H . c .,
$$

where $\mathrm{Y}$ is the symmetric neutralino mixing matrix ,its expression can be found in Ref. [15]. The masses of physical neutralinos can be obtained by diagonalizing $\mathrm{Y}$ by a unitary $5 \times 5$ matrix $N$

$$
m_{\chi_{i}^{0}} \delta_{i j}=N_{i m}^{*} Y_{m n} N_{j n}
$$

\section{THE SUSY-FCNC EFFECTS}

The full hadronic amplitudes for the kaon decays in Eq. (1) and $\Sigma \rightarrow p A_{1}^{0}$ all can be written in the following form

$$
\mathcal{M}_{\text {full }}=\mathcal{M}_{2 q}+\mathcal{M}_{4 q}
$$




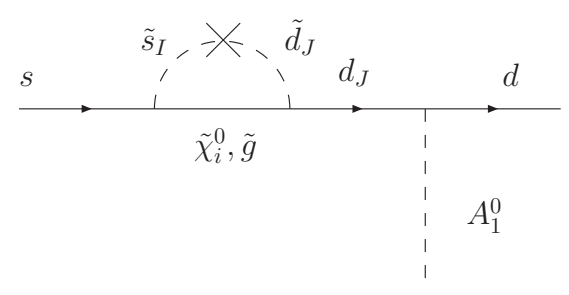

(a)

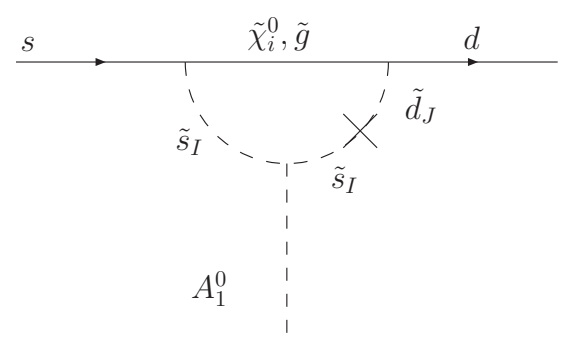

$(c)$

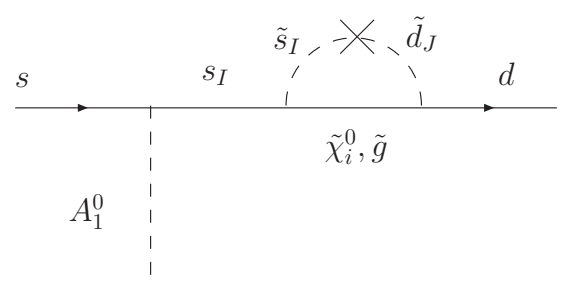

(b)

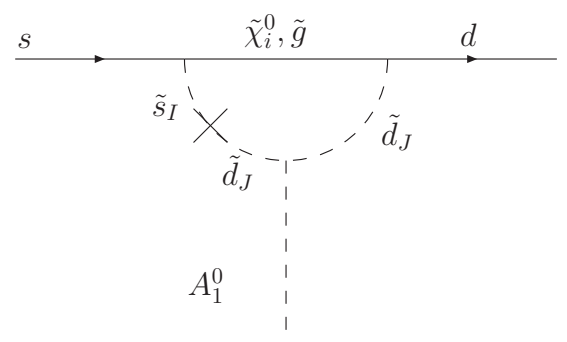

$(d)$

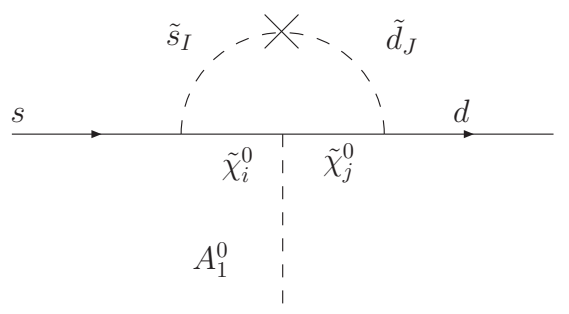

$(e)$

FIG. 1: Feynman diagrams for $|\Delta S|=1$ transitions, with I,J $=\mathrm{L}, \mathrm{R}$, and i,j $=1 \ldots 5$

where $\mathcal{M}_{2 q}$ arises from the SUSY-FCNC interactions at the quark level, and $\mathcal{M}_{4 q}$ arises from the four-quark interactions. Using the method shown in Ref. [8], we calculate the four-quark contributions, and their expressions and numerical results are given in Appendix B. In this section, we mainly concentrate on the hadronic amplitudes $\mathcal{M}_{2 q}$ from two-quark contribution and give our analytical results. In general, there are two kinds of FCNC contributions arising from neutralino and gluino exchange, respectively. The relevant Feynman diagrams for the $s \rightarrow d$ transitions are shown in Fig. 1. Note that there are five kinds of neutralinos in the loops in the NMSSM. Calculating these Feynman diagrams, one can obtain the two-quark FCNC Lagrangian for $s \rightarrow d A_{1}^{0}$

$$
\mathcal{L}_{\mathcal{A} s d}=i C_{L} \bar{d} \frac{1-\gamma_{5}}{2} s A_{1}^{0}+i C_{R} \bar{d} \frac{1+\gamma_{5}}{2} s A_{1}^{0}+\text { H.c. },
$$


with

$$
C_{L(R)}=C_{L(R)}^{\tilde{\chi}^{0}}+C_{L(R)}^{\tilde{g}}
$$

where $C_{L(R)}^{\tilde{\chi}^{0}}$ and $C_{L(R)}^{\tilde{g}}$ denote contributions from neutralino and gluino exchange, respectively. They are given by

$$
\begin{aligned}
C_{L}^{\tilde{\chi}^{0}}= & \frac{\alpha}{4 \pi} \sum_{i, j=1}^{5}\left\{\left(\delta_{12}^{d}\right)_{L L}\left[I_{i j}^{1} m_{d} L_{2 i} R_{2 j}^{*}-I_{i}^{3} m_{d} L_{2 i} R_{2 i}^{*}+I_{i}^{4} m_{d}\left(m_{s}^{2} L_{2 i} R_{1 i}^{*}-L_{1 i} R_{2 i}^{*}\right)\right]\right. \\
& +\left(\delta_{12}^{d}\right)_{L R}\left[I_{i j}^{1} m_{d} m_{s} L_{2 i} R_{1 j}^{*}-I_{i}^{3} m_{d} m_{s} L_{2 i} R_{1 i}^{*}-I_{i}^{4} m_{d} m_{s}\left(L_{1 i} R_{1 i}^{*}+L_{2 i} R_{2 i}^{*}\right)\right] \\
& +\left(\delta_{12}^{d}\right)_{R L}\left[I_{i j}^{1} L_{1 i} R_{2 j}^{*}-I_{i}^{3} L_{1 i} R_{2 i}^{*}+I_{i}^{4}\left(m_{d}^{2} L_{2 i} R_{2 i}^{*}+m_{s}^{2} L_{1 i} R_{1 i}^{*}\right)\right] \\
& \left.+\left(\delta_{12}^{d}\right)_{R R}\left[I_{i j}^{1} m_{s} L_{1 i} R_{1 j}^{*}-I_{i}^{3} m_{s} L_{1 i} R_{1 i}^{*}+I_{i}^{4} m_{s}\left(m_{d}^{2} L_{2 i} R_{1 i}^{*}-L_{1 i} R_{2 i}^{*}\right)\right]\right\} \\
C_{R}^{\tilde{\chi}^{0}}= & \frac{\alpha}{4 \pi} \sum_{i, j=1}^{5}\left\{\left(\delta_{12}^{d}\right)_{L L}\left[I_{i j}^{2} m_{s} R_{2 i} L_{2 j}^{*}+I_{i}^{3} m_{s} R_{2 i} L_{2 i}^{*}+I_{i}^{4} m_{s}\left(R_{2 i} L_{1 i}^{*}-m_{d}^{2} R_{1 i} L_{2 i}^{*}\right)\right]\right. \\
& +\left(\delta_{12}^{d}\right)_{L R}\left[I_{i j}^{2} R_{2 i} L_{1 j}^{*}+I_{i}^{3} R_{2 i} L_{1 i}^{*}-I_{i}^{4}\left(m_{d}^{2} R_{1 i} L_{1 i}^{*}+m_{s}^{2} R_{2 i} L_{2 i}^{*}\right)\right] \\
& +\left(\delta_{12}^{d}\right)_{R L}\left[I_{i j}^{2} m_{d} m_{s} R_{1 i} L_{2 j}^{*}+I_{i}^{3} m_{d} m_{s} R_{1 i} L_{2 i}^{*}+I_{i}^{4} m_{d} m_{s}\left(R_{2 i} L_{2 i}^{*}+R_{1 i} L_{1 i}^{*}\right)\right] \\
& \left.+\left(\delta_{12}^{d}\right)_{R R}\left[I_{i j}^{2} m_{d} R_{1 i} L_{1 j}^{*}+I_{i}^{3} m_{d} R_{1 i} L_{1 i}^{*}+I_{i}^{4} m_{d}\left(R_{2 i} L_{1 i}^{*}-m_{s}^{2} R_{1 i} L_{2 i}^{*}\right)\right]\right\} \\
C_{L}^{\tilde{g}}= & \frac{\alpha_{s}}{2 \pi} C_{F} m_{\tilde{g}}\left[2 V_{A d d} C_{1}\left(y_{\tilde{g}}\right)\left(\delta_{12}^{d}\right)_{R L}+V_{A \tilde{d} \tilde{d}} \frac{1}{m_{\tilde{d}}^{2}} D_{1}\left(y_{\tilde{g}}\right)\left(m_{d}\left(\delta_{12}^{d}\right)_{L L}+m_{s}\left(\delta_{s d}^{d}\right)_{R R}\right)\right], \\
C_{R}^{\tilde{g}}= & \frac{\alpha_{s}}{2 \pi} C_{F} m_{\tilde{g}}\left[2 V_{A d d} C_{1}\left(y_{\tilde{g}}\right)\left(\delta_{12}^{d}\right)_{L R}-V_{A \tilde{d} \tilde{d}} \frac{1}{m_{\tilde{d}}^{2}} D_{1}\left(y_{\tilde{g}}\right)\left(m_{d}\left(\delta_{12}^{d}\right)_{R R}+m_{s}\left(\delta_{s d}^{d}\right)_{L L}\right)\right],
\end{aligned}
$$

with

$$
\begin{aligned}
V_{A d d} & =\frac{g}{2 m_{W} \cos \beta} U_{11}^{P}, \\
V_{A \tilde{d} \tilde{d}} & =\frac{g}{2 m_{W} \cos \beta}\left[\lambda\left(v_{2} U_{13}^{P}+x U_{12}^{P}\right)-A_{D} U_{11}^{P}\right], \\
L_{1 i} & =\frac{2 N_{i, 1}^{*}}{3 \sqrt{2} c_{W}}, \\
L_{2 i} & =\frac{N_{i, 3}^{*}}{\sqrt{2} m_{W} s_{W} \cos \beta}, \\
R_{1 i} & =L_{2 i}^{*}, \\
R_{2 i} & =\frac{s_{W} N_{i, 1}-3 c_{W} N_{i, 2}}{3 \sqrt{2} c_{W} s_{W}}, \\
I_{i j}^{1} & =D_{2}\left(y_{i}, y_{j}\right) R_{1 i j}^{R^{\prime \prime}}+\frac{m_{\tilde{\chi}_{i}} m_{\tilde{\chi}_{j}}}{m_{\tilde{d}}^{2}} D_{2}\left(y_{i}, y_{j}\right) R_{1 i j}^{L^{\prime \prime}}, \\
I_{i j}^{2} & =D_{2}\left(y_{i}, y_{j}\right) R_{1 i j}^{L^{\prime \prime}}+\frac{m_{\tilde{\chi}_{i}} m_{\tilde{\chi}_{j}}}{m_{\tilde{d}}^{2}} D_{2}\left(y_{i}, y_{j}\right) R_{1 i j}^{R^{\prime \prime}}, \\
I_{i}^{3} & =2 V_{A d d} m_{\tilde{\chi}_{i}} C_{1}\left(y_{i}\right),
\end{aligned}
$$




$$
\begin{aligned}
I_{i}^{4} & =V_{A \tilde{d} \tilde{d}} \frac{m_{\tilde{\chi}_{i}}}{m_{\tilde{d}}^{2}} D_{1}\left(y_{i}\right), \\
y_{\tilde{g}} & =\frac{m_{\tilde{g}}^{2}}{m_{\tilde{d}}^{2}}, \quad y_{i}=\frac{m_{\tilde{\chi}_{i}^{0}}^{2}}{m_{\tilde{d}}^{2}}, i=1 \ldots 5,
\end{aligned}
$$

where $U^{P}$ is used to diagonalize the pseudoscalar Higgs mass matrices, the matrix $N_{i j}, i, j=$ $1 \cdots 5$ is the $5 \times 5$ unitary matrix defined in Sec II , $c_{W}=\cos \theta_{W}$, where $\theta_{W}$ is the Weinberg angle as usual, and

$$
\begin{aligned}
R_{1 i j}^{\prime \prime R}= & \frac{1}{2}\left[\left(U_{11}^{P} \cos \beta+U_{12}^{P} \sin \beta\right)\right. \\
& \times\left(\frac{g}{c_{W}}\left(N_{i 2} N_{j 3}^{*}+N_{j 2} N_{i 3}^{*}\right)-\sqrt{2} \lambda\left(N_{i 5} N_{j 4}^{*}+N_{j 5} N_{i 4}^{*}\right)\right) \\
& +\left(U_{11}^{P} \sin \beta-U_{12}^{P} \cos \beta\right) \\
& \left.\left(\frac{g}{c_{W}}\left(N_{i 2} N_{j 4}^{*}+N_{j 2} N_{i 4}^{*}\right)+\sqrt{2} \lambda\left(N_{i 5} N_{j 3}^{*}+N_{j 5} N_{i 3}^{*}\right)\right)\right] \\
& -\sqrt{2} k U_{13}^{P}\left(N_{i 5} N_{j 5}^{*}+N_{j 5} N_{i 5}^{*}\right) \\
R_{1 i j}^{\prime \prime R}= & -R_{1 i j}^{\prime \prime} .
\end{aligned}
$$

And the loop functions $C_{1}(x), D_{1}(x)$ and $D_{2}(x, y)$ are defined as

$$
\begin{aligned}
C_{1}(x) & =\frac{x-1-x \log (x)}{(1-x)^{2}}, \\
D_{1}(x) & =\frac{1-x^{2}+2 x \log (x)}{2(1-x)^{3}}, \\
D_{2}(x, y) & =-\frac{1}{(1-x)(1-y)}-\frac{x \log (x)}{(1-x)^{2}(x-y)}-\frac{y \log (y)}{(1-y)^{2}(y-x)} .
\end{aligned}
$$

The quark level effective Lagrangian in Eq.(13) can be mapped onto the chiral Lagrangian at the leading order[8]

$$
\mathcal{L}_{\mathcal{A}}=b_{D}\left\langle\bar{B}\left\{h_{A}, B\right\}\right\rangle+b_{F}\left\langle\bar{B}\left[h_{A}, B\right]\right\rangle+b_{0}\left\langle h_{A}\right\rangle\langle\bar{B} B\rangle+\frac{1}{2} f_{\pi}^{2} B_{0}\left\langle h_{A}\right\rangle+\text { H.c. },
$$

where

$$
B=\left(\begin{array}{ccc}
\frac{1}{\sqrt{2}} \Sigma^{0}+\frac{1}{\sqrt{6}} \Lambda & \Sigma^{+} & p \\
\Sigma^{-} & -\frac{1}{\sqrt{2}} \Sigma^{0}+\frac{1}{\sqrt{6}} \Lambda & n \\
\Xi^{-} & \Xi^{0} & -\frac{2}{\sqrt{6}} \Lambda
\end{array}\right)
$$

represents the baryon fields, $f_{\pi}=92.4 \mathrm{MeV}$ is the pion decay constant, $\langle\cdots\rangle \equiv \operatorname{Tr}(\cdots)$ in flavor- $S U(3)$. And

$$
h_{A}=-i\left(C_{R} \xi^{\dagger} h \xi^{\dagger}+C_{L} \xi h \xi\right) A_{1}^{0}
$$


where

$$
h=T_{6}+i T_{7}=\left(\begin{array}{ccc}
0 & 0 & 0 \\
0 & 0 & 1 \\
0 & 0 & 0
\end{array}\right)
$$

is used to specify the $s \rightarrow d$ transition,

$$
\xi=e^{i \pi / f_{\pi}}, \quad \Sigma=\xi \xi=e^{2 i \pi / f_{\pi}}
$$

and

$$
\pi=\frac{1}{\sqrt{2}}\left(\begin{array}{ccc}
\frac{1}{\sqrt{2}} \pi^{0}+\frac{1}{\sqrt{6}} \eta & \pi^{+} & K^{+} \\
\pi^{-} & -\frac{1}{\sqrt{2}} \pi^{0}+\frac{1}{\sqrt{6}} \eta & K^{0} \\
K^{-} & \bar{K}^{0} & -\frac{2}{\sqrt{6}} \eta
\end{array}\right)
$$

is the pion octet.

The two-quark amplitude $\mathcal{M}_{2 q}$ can be deduced from $\mathcal{L}_{\mathcal{A}}$ plus the usual chiral Lagrangian $\mathcal{L}_{s}$ for the strong interactions of hadrons, which is expressed as [32, 33, 34, 35]

$$
\begin{aligned}
\mathcal{L}_{s}= & i\left\langle\bar{B} \gamma^{\mu} D_{\mu} B\right\rangle-m_{0}\langle\bar{B} B\rangle+D\left\langle\bar{B} \gamma^{\mu} \gamma_{5}\left\{A_{\mu}, B\right\}\right\rangle+F\left\langle\bar{B} \gamma^{\mu} \gamma_{5}\left[A_{\mu}, B\right]\right\rangle \\
& +b_{D}\left\langle\bar{B}\left\{M_{+}, B\right\}\right\rangle+b_{F}\left\langle\bar{B}\left[M_{+}, B\right]\right\rangle+b_{0}\left\langle M_{+}\right\rangle\langle\bar{B} B\rangle \\
& +\frac{1}{4} f_{\pi}^{2}\left\langle\partial^{\mu} \Sigma^{\dagger} \partial_{\mu} \Sigma\right\rangle+\frac{1}{2} f_{\pi}^{2} B_{0}\left\langle M_{+}\right\rangle
\end{aligned}
$$

with

$$
\begin{gathered}
D^{\mu} B=\partial^{\mu} B+\left[V^{\mu}, B\right], \\
A^{\mu}=\frac{i}{2}\left(\xi \partial^{\mu} \xi^{\dagger}-\xi^{\dagger} \partial^{\mu} \xi\right), \quad M_{+}=\xi^{\dagger} M \xi^{\dagger}+\xi M^{\dagger} \xi, \\
V^{\mu}=\frac{1}{2}\left(\xi \partial^{\mu} \xi^{\dagger}+\xi^{\dagger} \partial^{\mu} \xi\right),
\end{gathered}
$$

where $M=\operatorname{diag}\left(\hat{m}, \hat{m}, m_{s}\right)$ is the quark mass matrix in the $m_{u}=m_{d}=\hat{m}$ limit.

Using the mass relations $m_{\Sigma}-m_{p}=2\left(b_{D}-b_{F}\right)\left(m_{s}-\hat{m}\right), m_{K}^{2}-m_{\pi}^{2}=B_{0}\left(m_{s}-\hat{m}\right)$ and $m_{K}^{2}=B_{0}\left(m_{s}+\hat{m}\right)$, the amplitudes for the different decay modes can be written as [8]

$$
\begin{aligned}
\mathcal{M}_{2 q}\left(K^{+} \rightarrow \pi^{+} A_{1}^{0}\right)= & -\sqrt{2} \mathcal{M}_{2 q}\left(K^{0} \rightarrow \pi^{0} A_{1}^{0}\right) \\
= & i\left(\frac{C_{L}+C_{R}}{2}\right) B_{0} \\
\mathcal{M}_{2 q}\left(\Sigma^{+} \rightarrow p A_{1}^{0}\right)= & i\left(\frac{C_{L}+C_{R}}{2}\right) \frac{B_{0}\left(m_{\Sigma}-m_{p}\right)}{m_{K}^{2}-m_{\pi}^{2}} \bar{p} \Sigma^{+} \\
& +i(D-F)\left(\frac{C_{L}-C_{R}}{2}\right) \frac{B_{0}\left(m_{\Sigma}+m_{p}\right)}{m_{K}^{2}-m_{A_{1}^{0}}^{2}} \bar{p} \gamma_{5} \Sigma^{+},
\end{aligned}
$$

where $B_{0}=2031 \mathrm{MeV}[4]$. 


\section{NUMERICAL RESULTS AND DISCUSSION}

In this section we present our numerical results. The Higgs sector of NMSSM is describe by the six independent parameters

$$
\lambda, \quad k, A_{\lambda}, A_{k}, \quad \tan \beta, \quad \mu,
$$

where $\mu=-\lambda x$. For convenience, we will take $m_{A_{1}^{0}}$ and the coupling of down-type quarks to $A_{1}^{0}, l_{d}$ instead of $\mathrm{k}$ and $\lambda$. We follow Ref. [7] to set $l_{d}=0.35,-\lambda x=150 \mathrm{GeV}$ and $\tan \beta=30$. $A_{k}$ and $A_{\lambda}$ are set as 0.001 and 0.002 , respectively. The mass of $A_{1}^{0}$ is set as $214.3 \mathrm{MeV}$ to satisfy the HyperCP data. We set the mass of gluino and average down type squark mass as $200 \mathrm{GeV}$ and $350 \mathrm{GeV}$, respectively. With our input, the mass of the neutralinos are around $100 \sim 800 \mathrm{GeV}$. Numerically, contributions from the exchange of the gluino and squarks are lager than contributions from the exchange of the neutralinos and squarks, i.e., $C_{L, R}^{\chi^{0}}$ are larger than $C_{L R}^{\tilde{g}}$ in most regions of parameter space. This is due to the effects of $\alpha_{s}$.

Our numerical results are shown in Figs, 3 - 7. We first assume that $\left(\delta^{12}\right)_{I J}$ are real. The allowed regions in parameter space are shown in Figs $\mathbf{3}$ - 5, where the grey areas are the allowed regions for $A_{1}^{0}$ to explain the HyperCP events. When the constraints obtained from the kaon decays are considered, the allowed parameter space are greatly reduced to the dark regions. From Figs - 5, the constraints on the combinations of the parameters can be obtained as following:

$$
\begin{aligned}
\left(\delta_{12}^{d}\right)_{L L(R R)}\left(\delta_{12}^{d}\right)_{L R(R L) \leq} \leq & 3.9 \times 10^{-12} \text { (without kaon bounds) } \\
& 3.7 \times 10^{-12} \text { (with kaon bounds) } \\
\left(\delta_{12}^{d}\right)_{L L}\left(\delta_{12}^{d}\right)_{R R} \leq & 3.9 \times 10^{-9} \text { (without kaon bounds) } \\
& 1.8 \times 10^{-14} \text { (with kaon bounds) } \\
\left(\delta_{12}^{d}\right)_{L R}\left(\delta_{12}^{d}\right)_{R L} \leq & 2.7 \times 10^{-16} \text { (without kaon bounds) } \\
& 0.9 \times 10^{-17} \text { (with kaon bounds) }
\end{aligned}
$$

It has been widely studied in the literature that the SUSY-FCNC effects has great impact on the $K^{0}-\bar{K}^{0}$ mixing if the relevant $\left(\delta_{12}^{d}\right)_{I J}$ are complex. So we further investigate the possible constraints on $\left(\delta_{12}^{d}\right)_{I J}$ from the $K_{L}-K_{S}$ mass difference $\Delta m_{K}$ and indirect CP violation parameter $\epsilon_{K}$. However, the constraints shown in Eq.(28) are roughly several orders smaller than those given in the literatures involving the SUSY-FCNC mediated $K^{0}-\bar{K}^{0}$ 
mixing [23, 24, 25, 26, 27, 28, 29], where $\left(\delta_{12}^{d}\right)_{L L}$ and $\left(\delta_{12}^{d}\right)_{L R(R L)}$ are around $\mathcal{O}\left(10^{-1} \sim 10^{-3}\right)$ and $\mathcal{O}\left(10^{-3} \sim 10^{-4}\right)$, respectively. This fact indicates that the constraints from the $K^{0}-\bar{K}^{0}$ mixing may be automatically satisfied once the constraints from $\Sigma \rightarrow p A_{1}^{0}$ and the rare kaon decays in Eq.(1) are satisfied. Our numerical results do indeed confirm that the constraints from the $K^{0}-\bar{K}^{0}$ mixing do not lead to more stringent constraints than those ones given from the kaon decays in Eq.(1) .

Figs $\left[6\right.$ and 7 show the constraints on the complex $\left(\delta_{12}^{d}\right)_{I J}$ from $\Sigma \rightarrow p A_{1}^{0}$ and rare kaon decays in Eq.(10). And the corresponding constraints on the combinations of parameters are given by

$$
\begin{aligned}
\operatorname{Re}\left(\delta_{12}^{d}\right)_{L R(R L)} \operatorname{Im}\left(\delta_{12}^{d}\right)_{L R(R L) \leq} \leq & 2.7 \times 10^{-16} \quad \text { (without kaon bounds), } \\
& 1.5 \times 10^{-18} \quad \text { (with kaon bounds), } \\
\operatorname{Re}\left(\delta_{12}^{d}\right)_{L L(R R)} \operatorname{Im}\left(\delta_{12}^{d}\right)_{L L(R R)} \leq & 1.8 \times 10^{-12} \text { (without kaon bounds), } \\
& 1.8 \times 10^{-14} \quad \text { (with kaon bounds) }
\end{aligned}
$$

From Figs 6] and 7, it can be seen that the grey areas are the allowed regions of the SUSYFCNC parameters for $A_{1}^{0}$ to explain the HyperCP events and the grey regions are greatly reduced to the dark ones when the constraints from rare kaon decays are considered. Even so, there are still regions in the SUSY-FCNC parameter space where $A_{1}^{0}$ in the NMSSM can be used to explain the HyperCP events without contradicting with the constraints from the rare kaon decays and the $K^{0}-\bar{K}^{0}$ mixing.

In conclusion, we have calculated the two-quark contributions to the decay $\Sigma^{+} \rightarrow p \mu^{+} \mu^{-}$ arising from the transition $s \rightarrow d A_{1}^{0}$ via the SUSY-FCNC couplings. Combining the twoquark contributions with the four-quark contributions, we show that there are regions in the SUSY-FCNC parameter space where the $A_{0}^{1}$ in the NMSSM can be identified with a new particle of mass $214.3 \mathrm{MeV}$, X, which can be used to explain the HyperCP events, while satisfying all the constraints from the measurements of the rare kaon decays. And once the constraints from the kaon decays are satisfied, the constraints from the $K^{0}-\bar{K}^{0}$ mixing are automatically satisfied. 


\section{Acknowledgments}

This work was supported in part by the National Natural Science Foundation of China, under Grant No. 10421503, No. 10575001 and No. 10635030, and the Key Grant Project of Chinese Ministry of Education under Grant No. 305001.

\section{Appendix A}

We give the Feynman rules used in our calculations in Fig 2 , where $V_{A \tilde{d} \tilde{d}}, V_{A d d}, L(R)_{1(2) i}$ and $R_{1 i j}^{L(R)^{\prime \prime}}$ are defined in Eq. (16), Eq. (17) and Eq. (201), respectively. And

$$
\delta_{I J}=\left\{\begin{array}{cc}
1 & I=J \\
0 & I \neq J
\end{array}\right.
$$

\section{Appendix B}

We collect expressions of the four-quark amplitudes for the different decay modes in this appendix. A detailed description can be found in Ref. [8], we cite their results here. As

pointed in Ref. [7], the couplings of $A_{1}^{0}$ to the up-type quarks tends to zero at the limit of large $\tan \beta$, so we neglect terms that are proportional to $l_{u}$.

The four-quark contributions for the kaon decays are as follow:

$$
\begin{aligned}
\mathcal{M}_{4 q}\left(K^{+} \rightarrow \pi^{+} A_{1}^{0}\right)= & i \frac{l_{d} \gamma_{8}}{v}\left\{-\frac{m_{\pi}^{2}}{2}+\left[\left(2 m_{K}^{2}+m_{\pi}^{2}-3 m_{A_{1}^{0}}^{2}\right) c_{\theta}-\sqrt{8}\left(m_{K}^{2}-m_{\pi}^{2}\right) s_{\theta}\right]\right. \\
& \times \frac{\left(4 m_{K}^{2}-3 m_{\pi}^{2}\right) c_{\theta}+\sqrt{2}\left(2 m_{K}^{2}-\tilde{m}_{0}^{2}\right) s_{\theta}}{6\left(m_{\eta}^{2}-m_{A_{1}^{0}}^{2}\right)}+\left[\left(2 m_{K}^{2}+m_{\pi}^{2}-3 m_{A_{1}^{0}}^{2}\right) s_{\theta}\right. \\
& \left.\left.+\sqrt{8}\left(m_{K}^{2}-m_{\pi}^{2}\right) c_{\theta}\right] \frac{\left(4 m_{K}^{2}-3 m_{\pi}^{2}\right) s_{\theta}-\sqrt{2}\left(2 m_{K}^{2}-\tilde{m}_{0}^{2}\right) c_{\theta}}{6\left(m_{\eta^{\prime}}^{2}-m_{A_{1}^{0}}^{2}\right)}\right\}, \\
\mathcal{M}_{4 q}\left(K^{0} \rightarrow \pi A_{1}^{0}\right)= & i \frac{l_{d} \gamma_{8}}{v}\left\{-\frac{\left(2 m_{K}^{2}-m_{\pi}^{2}-m_{A_{1}^{0}}^{2}\right) m_{\pi}^{2}}{\sqrt{8}\left(m_{A_{1}^{0}}^{2}-m_{\pi}^{2}\right)}\right. \\
& +\left[\left(2 m_{K}^{2}+m_{\pi}^{2}-3 m_{A_{1}^{0}}^{2}\right) c_{\theta}-\sqrt{8}\left(m_{K}^{2}-m_{\pi}^{2}\right) s_{\theta}\right] \\
& \times \frac{\left(4 m_{K}^{2}-3 m_{\pi}^{2}\right) c_{\theta}+\sqrt{2}\left(2 m_{K}^{2}-\tilde{m}_{0}^{2}\right) s_{\theta}}{6 \sqrt{2}\left(m_{A_{1}^{0}}^{2}-m_{\eta}^{2}\right)}+\left[\left(2 m_{K}^{2}+m_{\pi}^{2}-3 m_{A_{1}^{0}}^{2}\right) s_{\theta}\right. \\
& \left.\left.+\sqrt{8}\left(m_{K}^{2}-m_{\pi}^{2}\right) c_{\theta}\right] \frac{\left(4 m_{K}^{2}-3 m_{\pi}^{2}\right) s_{\theta}-\sqrt{2}\left(2 m_{K}^{2}-\tilde{m}_{0}^{2}\right) c_{\theta}}{6 \sqrt{2}\left(m_{A_{1}^{0}}^{2}-m_{\eta^{\prime}}^{2}\right)}\right\}
\end{aligned}
$$




$$
\begin{gathered}
\tilde{d}_{J} \\
----\times---- \\
-i\left(\Delta_{12}^{d}\right)_{I J}=-i\left(\delta_{12}^{d}\right)_{I J} m_{\tilde{d}}^{2}
\end{gathered}
$$

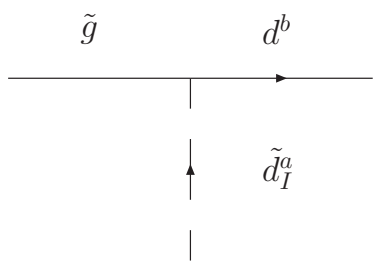

$i \sqrt{2} g_{s}\left(\delta_{I R} P_{L}-\delta_{I L} P_{R}\right) T_{b a}^{A}$

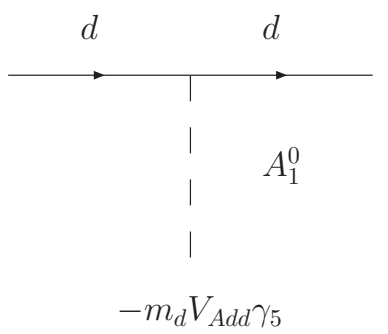

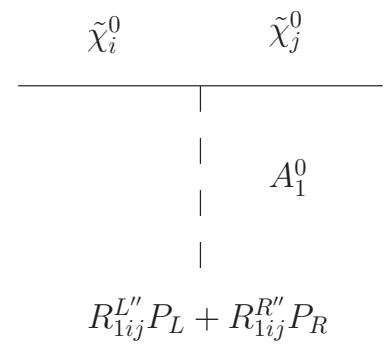
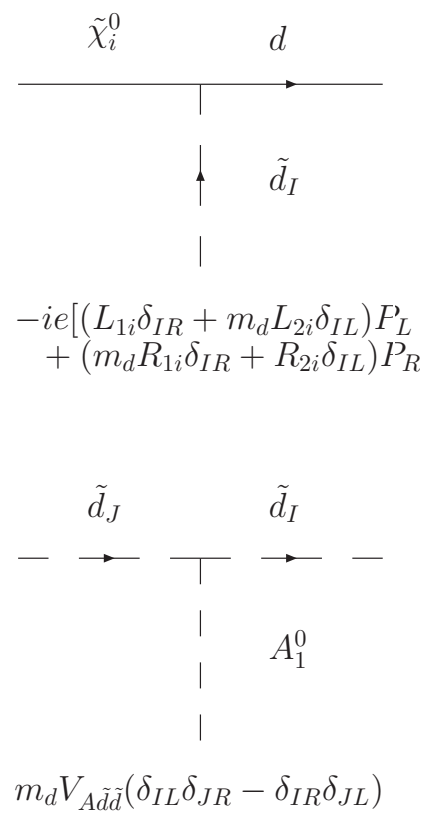

FIG. 2: Feynman rules used in our paper.

where $\gamma_{8}=-7.8 \times 10^{-8}, s_{\theta}$ and $c_{\theta}$ are short for $\sin \theta$ and $\cos \theta, \theta=-19.7^{\circ}$. And, the four-quark contributions to the $\Sigma \rightarrow p A_{1}^{0}$ process are

$$
\mathcal{M}_{4 q}\left(\Sigma^{+} \rightarrow p A_{1}^{0}\right)=i \bar{p}\left(A_{p A_{1}^{0}}-B_{p A_{1}^{0}} \gamma_{5}\right) \Sigma^{+}
$$

with

$$
\begin{aligned}
A_{p A_{1}^{0}}= & l_{d} \frac{f_{\pi}}{v} \frac{A_{p \pi^{0}}}{2}\left\{\frac{m_{\pi}^{2}}{m_{A_{1}^{0}}^{2}-m_{\pi}^{2}}+\frac{\left(4 m_{K}^{2}-3 m_{\pi}^{2}\right) c_{\theta}^{2}+\sqrt{2}\left(2 m_{K}^{2}-\tilde{m}_{0}^{2}\right) c_{\theta} s_{\theta}}{m_{\eta}^{2}-m_{A_{1}^{0}}^{2}}\right. \\
& \left.+\frac{\left(4 m_{K}^{2}-3 m_{\pi}^{2}\right) s_{\theta}^{2}-\sqrt{2}\left(2 m_{K}^{2}-\tilde{m}_{0}^{2}\right) c_{\theta} s_{\theta}}{m_{\eta^{\prime}}^{2}-m_{A_{1}^{0}}^{2}}\right\}
\end{aligned}
$$


and

$$
\begin{aligned}
B_{p A_{1}^{0}}= & l_{d} \frac{f_{\pi}}{v} \frac{B_{p \pi^{0}}}{2}\left\{\frac{m_{\pi}^{2}}{m_{A_{1}^{0}}^{2}-m_{\pi}^{2}}+\frac{\left(4 m_{K}^{2}-3 m_{\pi}^{2}\right) c_{\theta}^{2}+\sqrt{2}\left(2 m_{K}^{2}-\tilde{m}_{0}^{2}\right) c_{\theta} s_{\theta}}{m_{\eta}^{2}-m_{A_{1}^{0}}^{2}}\right. \\
& \left.+\frac{\left(4 m_{K}^{2}-m_{\pi}^{2}\right) s_{\theta}^{2}-\sqrt{2}\left(2 m_{K}^{2}-\tilde{m}_{0}^{2}\right) c_{\theta} s_{\theta}}{m_{\eta^{\prime}}^{2}-m_{A_{1}^{0}}^{2}}\right\},
\end{aligned}
$$

where $A_{p \pi^{0}}=-3.25 \times 10^{-7}, B_{p \pi^{0}}=26.67 \times 10^{-7}$.

Numerically, the above amplitudes are

$$
\begin{aligned}
\mathcal{M}_{4 q}\left(\Sigma \rightarrow p A_{1}^{0}\right) & =i \bar{p}\left(-6.96 \times 10^{-7} l_{d} \frac{f_{\pi}}{v}-\left(5.71 \times 10^{-6}\right) l_{d} \frac{f_{\pi}}{v} \gamma_{5}\right) \Sigma^{+}, \\
\mathcal{M}_{4 q}\left(K^{+} \rightarrow \pi^{+} A_{1}^{0}\right) & =-i 1.08 \times 10^{-7} l_{d} \frac{m_{K}^{2}}{v} \\
\mathcal{M}_{4 q}\left(K^{0} \rightarrow \pi A_{1}^{0}\right) & =i 1.12 \times 10^{-7} l_{d} \frac{m_{K}^{2}}{v} .
\end{aligned}
$$

[1] D. S. Gorbunov and V. A. Rubakov, Phys. Rev. D73, 035002 (2006), hep-ph/0509147.

[2] C. Q. Geng and Y. K. Hsiao, Phys. Lett. B632, 215 (2006), hep-ph/0509175.

[3] N. G. Deshpande, G. Eilam, and J. Jiang, Phys. Lett. B632, 212 (2006), hep-ph/0509081.

[4] X.-G. He, J. Tandean, and G. Valencia, Phys. Lett. B631, 100 (2005), hep-ph/0509041.

[5] E. C. Dukes (HyperCP) (2006), hep-ex/0606038.

[6] S. V. Demidov and D. S. Gorbunov, JETP Lett. 84, 479 (2007), hep-ph/0610066.

[7] X.-G. He, J. Tandean, and G. Valencia, Phys. Rev. Lett. 98, 081802 (2007), hep-ph/0610362.

[8] X.-G. He, J. Tandean, and G. Valencia, Phys. Rev. D74, 115015 (2006), hep-ph/0610274.

[9] C.-H. Chen, C.-Q. Geng, and C.-W. Kao (2007), arXiv:0708.0937 [hep-ph].

[10] M. L. Mangano and P. Nason, Mod. Phys. Lett. A22, 1373 (2007), arXiv:0704.1719 [hep-ph].

[11] H. Park et al. (HyperCP), Phys. Rev. Lett. 94, 021801 (2005), hep-ex/0501014.

[12] X.-G. He, J. Tandean, and G. Valencia, Phys. Rev. D72, 074003 (2005), hep-ph/0506067.

[13] M. Drees, Int. J. Mod. Phys. A4, 3635 (1989).

[14] J. R. Ellis, J. F. Gunion, H. E. Haber, L. Roszkowski, and F. Zwirner, Phys. Rev. D39, 844 (1989).

[15] F. Franke and H. Fraas, Int. J. Mod. Phys. A12, 479 (1997), hep-ph/9512366.

[16] H. Ma et al. (e865), Phys. Rev. Lett. 84, 2580 (2000), hep-ex/9910047. 
[17] H. K. Park et al. (HyperCP), Phys. Rev. Lett. 88, 111801 (2002), hep-ex/0110033.

[18] J. R. Batley et al. (NA48/1), Phys. Lett. B599, 197 (2004), hep-ex/0409011.

[19] B. Aubert et al. (BABAR), Phys. Rev. Lett. 93, 081802 (2004), hep-ex/0404006.

[20] M. Iwasaki et al. (Belle), Phys. Rev. D72, 092005 (2005), hep-ex/0503044.

[21] L. J. Hall, V. A. Kostelecky, and S. Raby, Nucl. Phys. B267, 415 (1986).

[22] F. Gabbiani and A. Masiero, Nucl. Phys. B322, 235 (1989).

[23] J. S. Hagelin, S. Kelley, and T. Tanaka, Nucl. Phys. B415, 293 (1994).

[24] F. Gabbiani, E. Gabrielli, A. Masiero, and L. Silvestrini, Nucl. Phys. B477, 321 (1996), hep-ph/9604387.

[25] J. S. Hagelin, S. Kelley, and T. Tanaka, Mod. Phys. Lett. A8, 2737 (1993), hep-ph/9304218.

[26] J. A. Bagger, K. T. Matchev, and R.-J. Zhang, Phys. Lett. B412, 77 (1997), hep-ph/9707225.

[27] M. Ciuchini et al., Nucl. Phys. B523, 501 (1998), hep-ph/9711402.

[28] M. Ciuchini et al., JHEP 10, 008 (1998), hep-ph/9808328.

[29] D. Becirevic and G. Villadoro, Phys. Rev. D70, 094036 (2004), hep-lat/0408029.

[30] G. Hiller, Phys. Rev. D70, 034018 (2004), hep-ph/0404220.

[31] B. A. Dobrescu and K. T. Matchev, JHEP 09, 031 (2000), hep-ph/0008192.

[32] J. Gasser and H. Leutwyler, Ann. Phys. 158, 142 (1984).

[33] J. Bijnens, H. Sonoda, and M. B. Wise, Nucl. Phys. B261, 185 (1985).

[34] E. E. Jenkins, Nucl. Phys. B375, 561 (1992).

[35] E. E. Jenkins and A. V. Manohar (1991), talk presented at the Workshop on Effective Field Theories of the Standard Model, Dobogoko, Hungary, Aug 1991. 


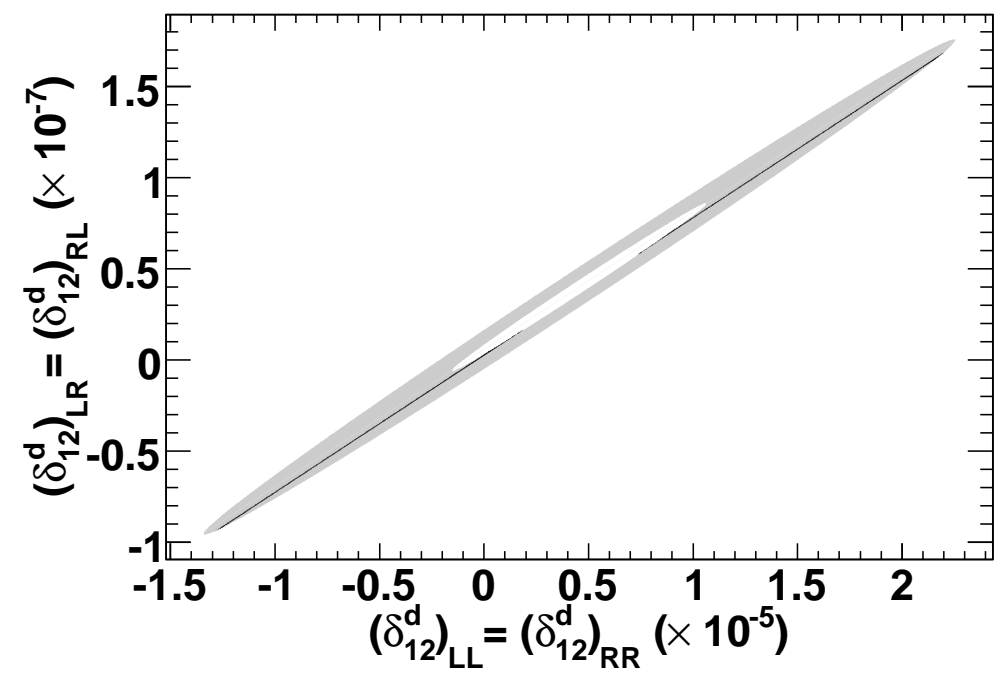

FIG. 3: The allowed values of $\left(\delta_{12}^{d}\right)_{L R}=\left(\delta_{12}^{d}\right)_{R L}$ as a function of $\left(\delta_{12}^{d}\right)_{L L}=\left(\delta_{12}^{d}\right)_{R R}$. The grey area is the regions where $A_{1}^{0}$ can explain the HyperCP events, when the constraints from rare kaon decays are considered, the allowed regions are greatly reduced to the dark ones.

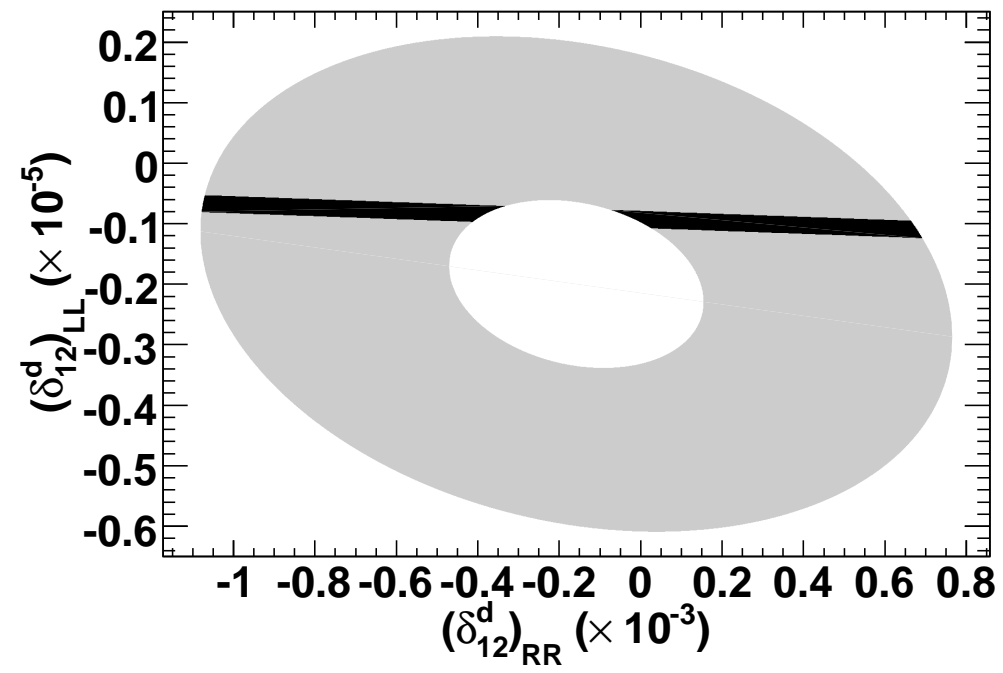

FIG. 4: The allowed values of $\left(\delta_{12}^{d}\right)_{L L}$ as a function of $\left(\delta_{12}^{d}\right)_{R R}$, The grey area is the regions where $A_{1}^{0}$ can explain the HyperCP events, when the constraints from rare kaon decays are considered, the allowed regions are greatly reduced to the dark ones. 


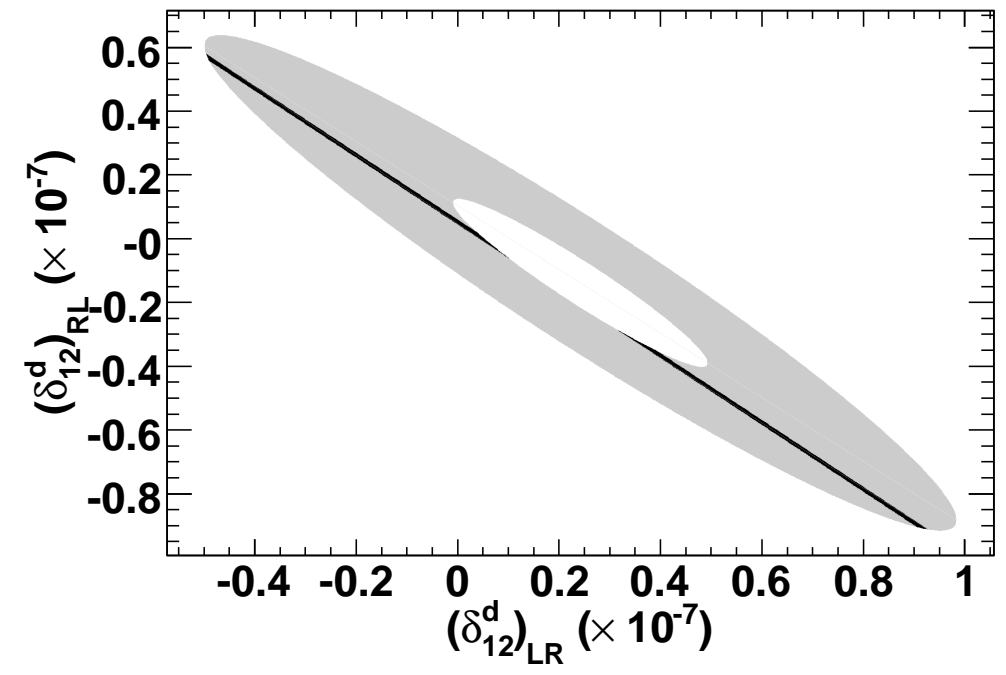

FIG. 5: The allowed values of $\left(\delta_{12}^{d}\right)_{R L}$ as a function of $\left(\delta_{12}^{d}\right)_{L R}$, The grey area is the regions where $A_{1}^{0}$ can explain the HyperCP events, when the constraints from rare kaon decays are considered, the allowed regions are greatly reduced to the dark ones.

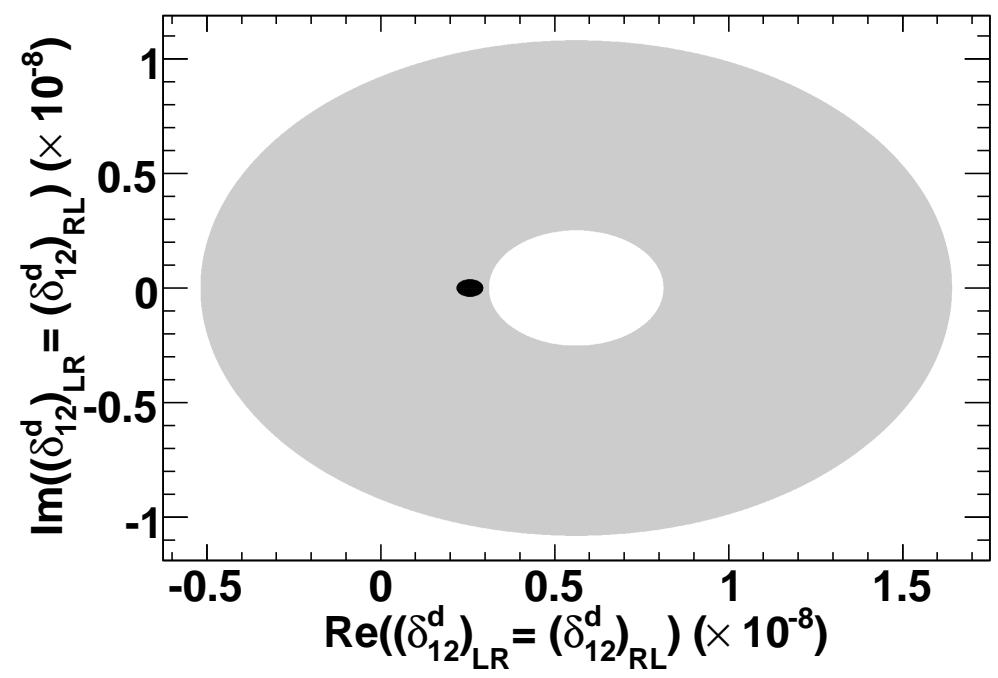

FIG. 6: The allowed values of $\operatorname{Im}\left\{\left(\delta_{12}^{d}\right)_{L R}=\left(\delta_{12}^{d}\right)_{R L}\right\}$ as a function of $\operatorname{Re}\left\{\left(\delta_{12}^{d}\right)_{L R}=\left(\delta_{12}^{d}\right)_{R L}\right\}$. The grey regions denotes the survival regions for explaining the HyperCP events alone; In the dark regions, the $A_{1}^{0}$ can explain the HyperCP events and simultaneously satisfy the bounds originate kaon decays and the $K^{0}-\bar{K}^{0}$ mixing. 


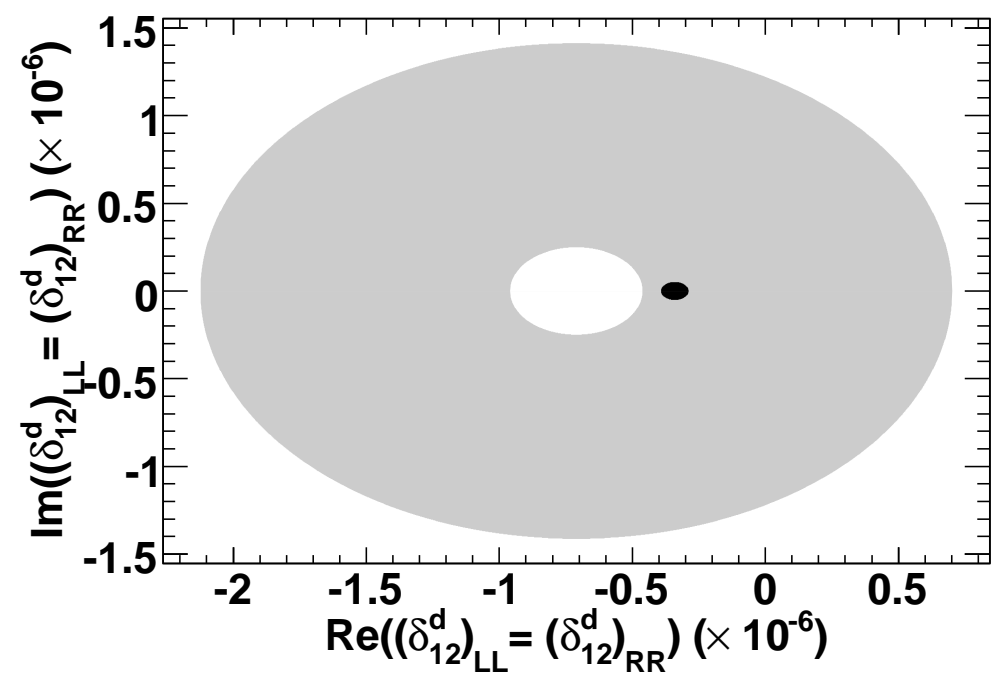

FIG. 7: The allowed values of $\operatorname{Im}\left\{\left(\delta_{12}^{d}\right)_{L L}=\left(\delta_{12}^{d}\right)_{R R}\right\}$ as a function of $\operatorname{Re}\left\{\left(\delta_{12}^{d}\right)_{L L}=\left(\delta_{12}^{d}\right)_{R R}\right\}$. The grey regions denotes the survival regions for explaining the HyperCP events alone; In the dark regions, the $A_{1}^{0}$ can explain the HyperCP events and simultaneously satisfy the bounds originate kaon decays and the $K^{0}-\bar{K}^{0}$ mixing. 